\title{
Reconstruction of the hepatic artery using the superior mesenteric artery for liver transplantation
}

\author{
Jiali Fang ${ }^{1}$, Zheng Chen ${ }^{1}$, Nan Jiang ${ }^{2}$, Qiang $\mathrm{He}^{3}$, Shaoping Wang ${ }^{4}$, Weiting Zhang ${ }^{1}$, Junjie Ma ${ }^{1}$, \\ Guanghui Li ${ }^{1}$, Lei Zhang ${ }^{1}$, Lu Xu ${ }^{1}$, Wei Yin ${ }^{1}$, Xingqiang Lai ${ }^{1}$, Rongxin Chen ${ }^{1}$, Li Li ${ }^{1}$, Luhao Liu ${ }^{1}$, \\ Yunyi Xiong ${ }^{1}$, Tao Zhang ${ }^{1}$, Hailin Xu ${ }^{1}$, Jiao Wan ${ }^{1}$, Jialin Wu ${ }^{1}$, Guanghui Pan ${ }^{1}$, Bingyi Shi ${ }^{5}$, Kun Li $^{6}$ \\ ${ }^{1}$ Organ Transplant Center, Second Affiliated Hospital of Guangzhou Medical University, Guangzhou, China; ${ }^{2}$ Liver Transplant Center, The Third \\ People's Hospital of Shenzhen, Shenzhen, China; ${ }^{3}$ Liver Transplant Center, Beijing Chao-Yang Hospital, Capital Medical University, Beijing, China; \\ ${ }^{4}$ Department of Hepatobiliary Surgery, General Hospital of Southern Theater Command, Guangzhou, China; ${ }^{5}$ Organ Transplantation Research \\ Institute, The 8th Medical Center of Chinese PLA General Hospital, Beijing, China; ${ }^{6}$ Department of Hepatobiliary Surgery, The Second People's \\ Hospital of Guiyang, Guiyang, China \\ Contributions: (I) Conception and design: J Fang; (II) Administrative support: Z Chen, W Zhang, J Ma; (III) Provision of study materials or patients: \\ Z Chen, N Jiang, Q He, S Wang, K Li; (IV) Collection and assembly of data: N Jiang, Q He, S Wang, K Li, L Liu, Y Xiong, T Zhang, H Xu, J Wan, \\ J Wu; (V) Data analysis and interpretation: J Fang, G Pan, B Shi, G Li, L Zhang, L Xu, W Yin, X Lai, R Chen, L Li; (VI) Manuscript writing: All \\ authors; (VII) Final approval of manuscript: All authors. \\ Correspondence to: Zheng Chen. Organ Transplant Center, Second Affiliated Hospital of Guangzhou Medical University, No. 250, Changgang East \\ Road, Haizhu District, Guangzhou 510260, China. Email: docchenzheng@163.com; Kun Li. The Second People’s Hospital of Guiyang, 547 Jinyang \\ South Road, Guanshanhu District, Guiyang 550023, China. Email: 386801779@qq.com.
}

Background: To investigate the application of the superior mesenteric artery (SMA) for the in vitro reconstruction of the hepatic artery for liver transplantation, and to improve the success rate and safety of donor liver transplantation.

Methods: The donor liver and the pancreas were obtained, and the SMA and its branches were used to reconstruct the hepatic artery. Liver transplantation was performed after reconstruction to understand the intraoperative situation after donor liver opening, as well as postoperative liver function. Color Doppler ultrasound of the transplanted liver was also performed.

Results: During the period from September 2016 to March 2020, a total of 98 pancreases were obtained. The common hepatic artery and gastroduodenal artery loop (CHA-GDA) were preserved to the donor pancreas, and only the proper hepatic artery (PHA) or left/right hepatic artery (LHA/RHA) were preserved to the donor liver. If the PHA of the donor liver was short or absent, the SMA was used for lengthening the PHA or in vitro reconstruction of the LHA/RHA, followed by implantation of the donor liver after reconstruction. A total of 17 cases of this type of donor liver required mesenteric artery lengthening or reconstruction. After opening, the donor liver was well-filled, bile secretion was normal, and liver function recovered as scheduled after surgery. Color Doppler ultrasound and CT angiography (CTA) of the transplanted liver revealed that hepatic arteries were normal without complications such as hepatic artery embolism.

Conclusions: In vitro reconstruction of the hepatic artery with the SMA is an effective new method of vascular reconstruction, which ensures the blood flow of the hepatic artery, reduces the anastomosis difficulty of the arteries of the donor liver, and reduces the occurrence of vascular complications.

Keywords: Donor liver; superior mesenteric artery (SMA); proper hepatic artery (PHA); common hepatic artery (CHA); artery reconstruction

Submitted Sep 23, 2020. Accepted for publication Nov 16, 2020.

doi: $10.21037 /$ atm-20-7200

View this article at: http://dx.doi.org/10.21037/atm-20-7200 


\section{Page 2 of 7}

\section{Introduction}

Simultaneous pancreas and kidney transplantation is the most effective treatment option for end-stage diabetic nephropathy, which can eradicate diabetes mellitus as well as renal failure, and improve the long-term survival rate of diabetic patients compared to renal transplantation alone. However, the integrity of the common hepatic artery-pancreaticoduodenal artery (CHA-PDA) needs to be preserved during pancreatic repair in simultaneous pancreas and kidney transplantation to ensure the blood flow of the duodenum, and to reduce intestinal complications after simultaneous pancreas and kidney transplantation/pancreas transplantation. Therefore, the only arteries left to the donor liver are the proper hepatic artery (PHA) or the left/right hepatic artery (LHA/RHA), which often increases the difficulty of liver transplantation and requires revascularization in some cases. In previous experience, the donor's iliac artery/iliac vein were used for reconstruction (1). Our center used the superior mesenteric artery (SMA) and its branches to reconstruct the PHA and the LHA/RHA, and achieved good results.

We present the following article in accordance with the STROBE reporting checklist (available at http://dx.doi. org/10.21037/atm-20-7200).

\section{Methods}

All procedures performed in this study involving human participants were in accordance with the Declaration of Helsinki (as revised in 2013). The study was approved by the Second Affiliated Hospital of Guangzhou Medical University (No. ChiCTR1900026543) and written informed consent was obtained from all patients.

\section{Patient characteristics}

Of the 17 donors, 9 had severe traumatic brain injury and 8 had cerebral hemorrhage. Donors were aged $20-51$ years, 15 were male and 2 were female, with BMI ranging between 18.75-27.72. Total bilirubin ranged between 11.4-119.2 umol/L (median $33.7 \mu \mathrm{mol} / \mathrm{L}$ ), alanine aminotransferase (ALT) ranged between $15-134 \mu / \mathrm{L}$ (median $32 \mu / \mathrm{L}$ ), aspartate aminotransferase (AST) ranged between 19-98 $\mu / \mathrm{L}$ (median $45 \mu / \mathrm{L}$ ), and gammaglutamyl transferase (GGT) ranged between 20-237 $\mu / \mathrm{L}$ (median $57 \mu / \mathrm{L})$.

The primary diseases of recipients were chronic acute liver failure in 5 cases, primary liver cancer in 5 cases, and chronic liver failure in 7 cases. The model for end-stage liver disease (MELD) scores (7-40 points) and Child-Pugh

\section{Fang et al. Reconstruction of the hepatic artery for transplantation}

grades (A-C) were also evaluated.

\section{Surgery process}

After routine en bloc acquisition of the donor liver, pancreas, and both kidneys, the abdominal aorta was cut between the renal artery and the SMA, the inferior vena cava was transected below the liver and above the right renal vein, the donor pancreas and liver were preserved, and the renal junction kidney group was trimmed. The surrounding tissues, including lymphatic vessels, the hepatic plexus, and surrounding connective tissue, were carefully dissected at the hepatic pedicle at the first porta hepatis. The common bile duct, hepatic portal vein, and PHA were freed from the right to the left, respectively. The distal part of the common bile duct was transected and doubly ligated with no. 7 and 4 silk sutures, the hepatic portal vein was transected approximately $1.5-2 \mathrm{~cm}$ away from the outlet of the pancreas, and the PHA was freed in the direction of the pancreas. The common hepatic artery and gastroduodenal arterial arch could be observed, the arterial variation was explored, then the integrity of the arterial arch was obtained. The PHA was transected approximately $0.5 \mathrm{~cm}$ away from the distal end of the arterial arch, the PHA transection was closed with a 5-0 atraumatic vascular suture, and the opening of the donor liver end was examined (Figure 1). If the hepatic artery was variant, the variant hepatic artery branches were divided separately (Figures 2 and 3). The SMA and its branches were obtained for liver reserve (Figure 4).

The donor liver was trimmed in vitro and revascularization was performed. After trimming the SMA and its branches and selecting the appropriate branches, the hepatic artery and SMA branches were end-to-end sutured with appropriate atraumatic vascular sutures, fixed at 4 points, then intermittently sutured. The reconstructed artery was then anastomosed end-to-end with the hepatic artery in vivo (Figures 5 and 6 ). If the PHA of the donor liver was not variable but short, end-to-end anastomosis was performed with the SMA and the PHA of the donor liver in vitro, and end-to-end anastomosis was performed between the SMA of the donor and the PHA of the recipient liver after the portal vein was opened (Figure 7).

\section{Statistical analysis}

SPSS20.0 software was used to process the data, and the measurement data of non-normal distribution was represented by the median. 


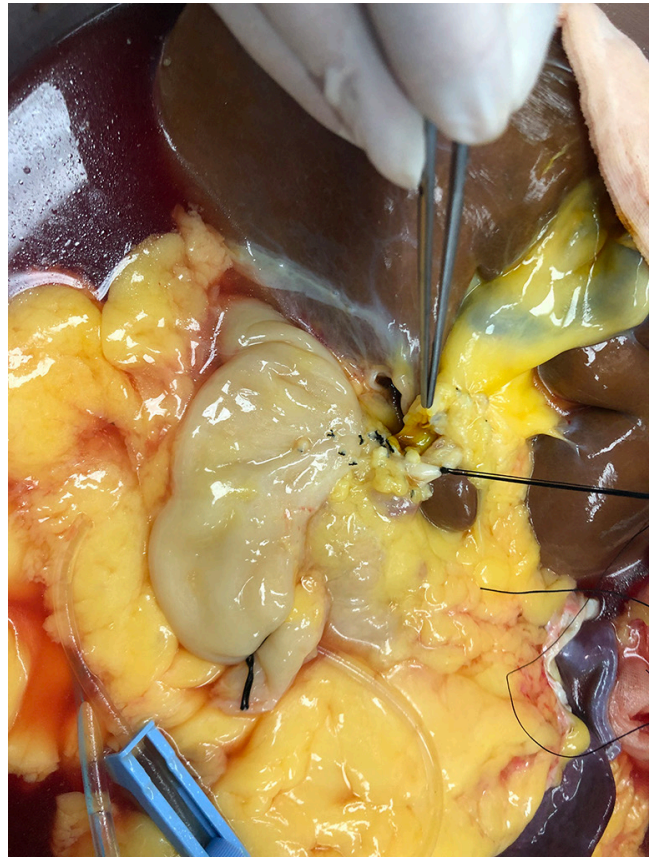

Figure 1 The opening of the proper hepatic artery, and the left and right hepatic artery with the common trunk.

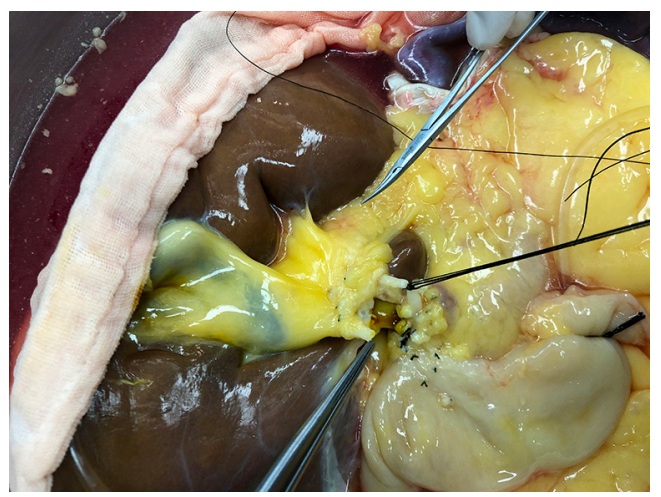

Figure 2 No common proper hepatic artery opening between the left and right hepatic arteries of the donor liver.

\section{Results}

Of the 17 recipients, 14 were male and 3 were female, aged between 27 to 56 years. The MELD scores ranged from 1 to 35 , and the Child-Pugh classifications were A to C. A total of 15 cases were modified piggyback liver transplantations, and 2 cases were classical piggyback liver transplantations. The intra-operative hemorrhagic volume was $300-6,000 \mathrm{~mL}$, blood transfusion volume was $800-10,955 \mathrm{~mL}$, warm ischemia time was $0 \mathrm{~min}$, cold ischemia time was $5-8$ hours,

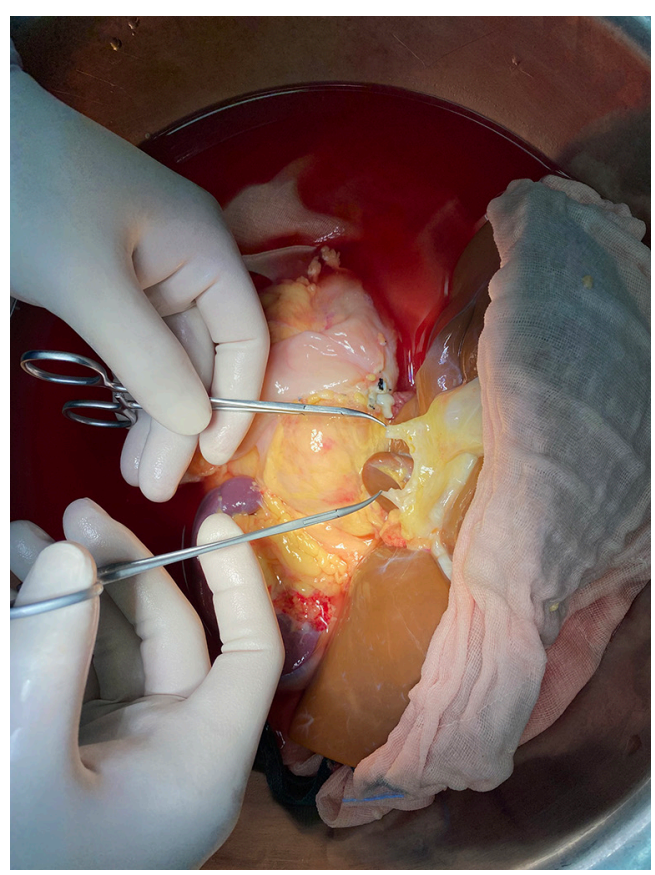

Figure 3 No common proper hepatic artery opening between the left and right hepatic arteries of the donor liver.

and the anhepatic period was 32-57 min, with an average of $44.5 \mathrm{~min}$. The hospital stays ranged between 34-120 days, with an average of 61.3 days, and the ICU stays ranged between 1-8 days, with an average of 2.83 days. The patients were followed up for 1-96 months, and the prognosis was good. The color Doppler ultrasound of the transplanted liver showed that there were no obvious abnormalities of the blood flow signal of the hepatic artery. The computed tomography angiography (CTA) of the transplanted liver showed that the direction of the transplanted hepatic artery was normal, and the blood flow filling was normal (Figure 8). Postoperative bilirubin and transaminase levels decreased steadily (Figures 9-11). One patient developed hepatic artery stenosis (HAS) 3 months after surgery, and the stenotic segment disappeared after stent placement, whilst all other patients had no vascular complications. All patients had no biliary complications, and no acute/chronic rejection occurred.

\section{Discussion}

Good arterial perfusion is important for maintaining the function of the transplanted liver and reducing biliary complications. The incidence of hepatic artery complications has been reported in the literature to range from $2 \%$ to $9 \%$ in adult recipients and $7 \%$ to $26 \%$ in 

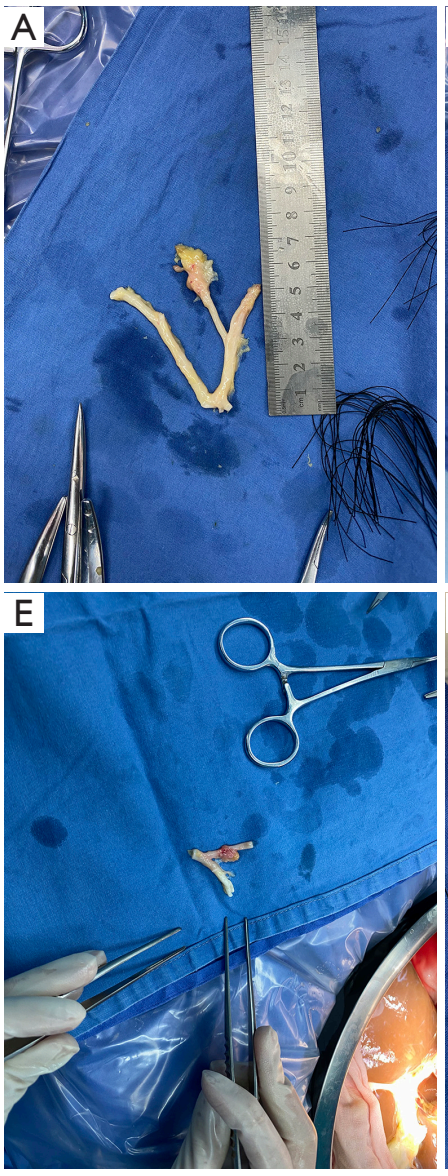
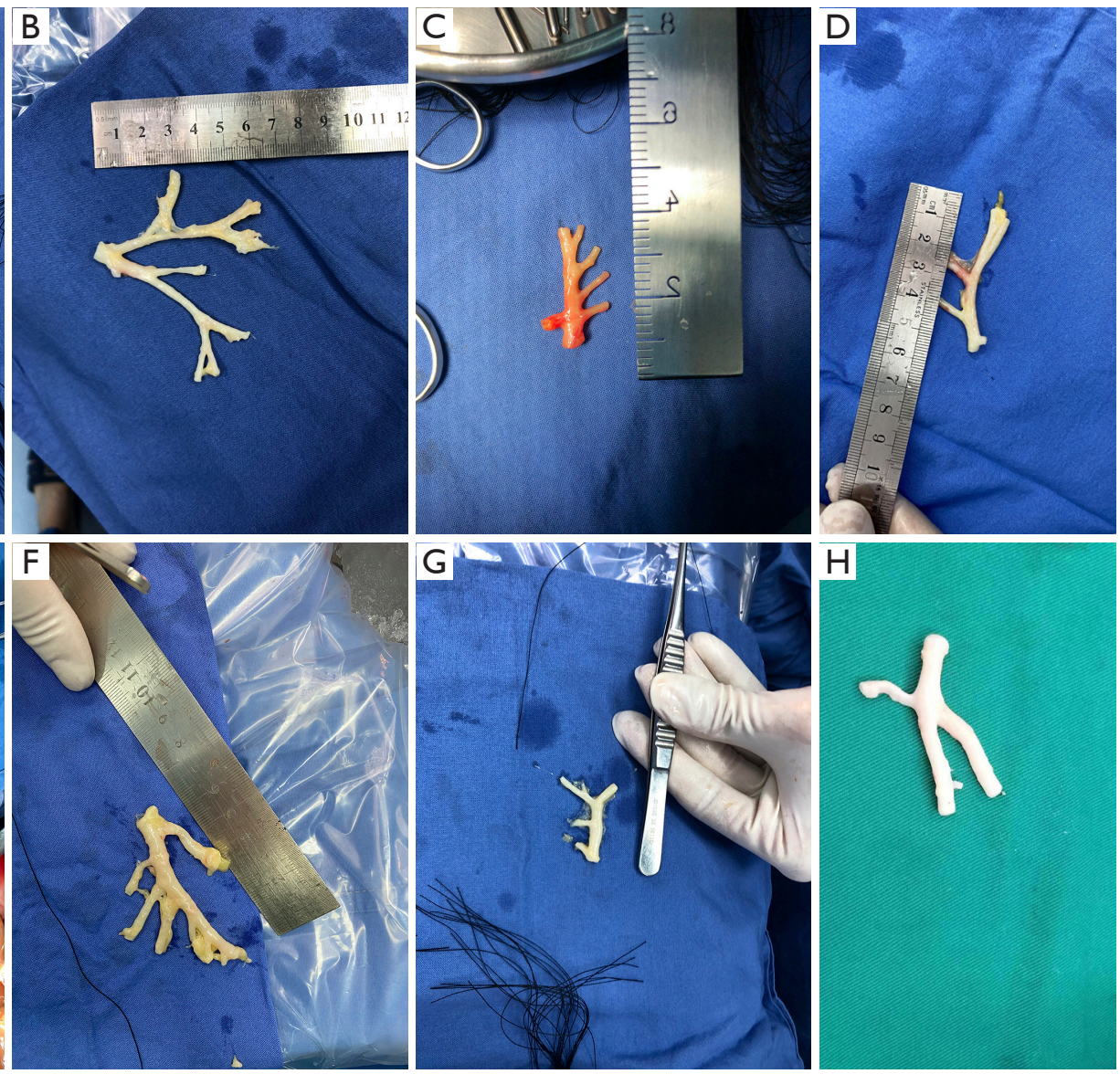

Figure 4 Superior mesenteric artery and its branches.

pediatric recipients $(1,2)$. They mainly include hepatic artery thrombosis (HAT), HAS, as well as pseudoaneurysms $(3,4)$. The hepatic arterial system is the most important source of the intrahepatic and extrahepatic biliary system and anastomosis in recipients. Therefore, abnormal hepatic artery hemodynamics after liver transplantation will directly lead to ischemic-type biliary lesions (ITBL), significantly increasing the occurrence of fatal biliary complications. Hernandez et al. (5) reported that out of 300 cases of liver transplantation, 12 cases had biliary stricture with patent hepatic artery without re-transplantation, while 9 cases had biliary tree necrosis or biliary stricture caused by HAT or HAS requiring re-transplantation. Torras et al. (6) reported that 16 out of 22 cases $(72.7 \%)$ of ischemic biliary stricture required re-transplantation. Therefore, abnormal hepatic artery hemodynamics are independent risk factors for biliary complications.

It is generally accepted that the surgical suture technique is an important risk factor leading to HAT (7), which is also related to immune factors, donor liver quality, ischemic time, infection, and recipient coagulation status $(8,9)$. Yang et al. (10) reported that complex graft hepatic artery reconstruction is also one of the risk factors for HAT.

The external iliac artery of the donor has been predominantly used in previous hepatic artery or branch reconstructions, however, it increases the incidence of HAT after pulse surgery (11). Del Gaudio et al. (12) reported that the incidence of postoperative HAT was $21.8 \%$ and $8.6 \%$ in recipients who had donor iliac artery bypass to reconstruct the hepatic artery and those who did not, respectively, whilst Warner et al. (13) reported rates of $11.1 \%$ and $4.4 \%$, respectively. We used the donor SMA to reconstruct the hepatic artery, which produced a high success rate. Furthermore, we chose to reconstruct in vitro, thus reducing the surgical difficulty of in vivo reconstruction. Our results showed that $5.9 \%(1 / 17)$ of recipients with hepatic artery reconstruction using the SMA developed HAS after surgery, all of which were mild and did not affect liver function. Additionally, no arterial embolism occurred, which is comparable to the incidence of HAT in non-bypass recipients 

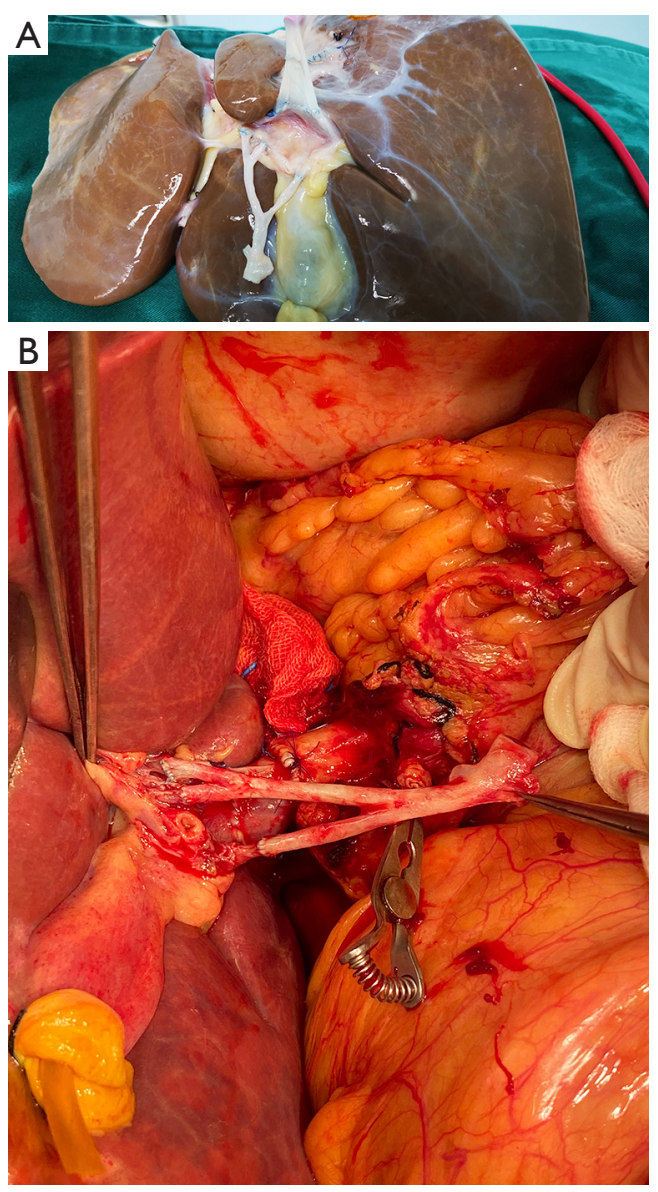

Figure 5 Reconstruction of hepatic artery using superior mesenteric artery and opening the hepatic portal vein. (A) Branches of hepatic artery anastomoses with branches of the superior mesenteric artery. (B) After opening the hepatic portal vein, the superior mesenteric artery was end-to-end anastomosed to the recipient hepatic artery.

reported in the previous literature $(12,13)$. Therefore, our transplantation method was effective and safe.

The SMA has distinct anatomical advantages. It is an important branch of the abdominal aorta, arising from the anterior wall of the abdominal aorta between the 12th thoracic vertebra and the 2 nd lumbar vertebral body, with an origin approximately $10 \mathrm{~mm}$ below the celiac artery and located behind the pancreas. The SMA trunk descends posterior to the pancreaticocervicopancreatic junction, accompanies the superior mesenteric vein, and passes between the lower edge of the pancreatic neck and the duodenum. It crosses down to the level of the duodenum into the root of the small bowel mesentery. Xu and Tang (14)
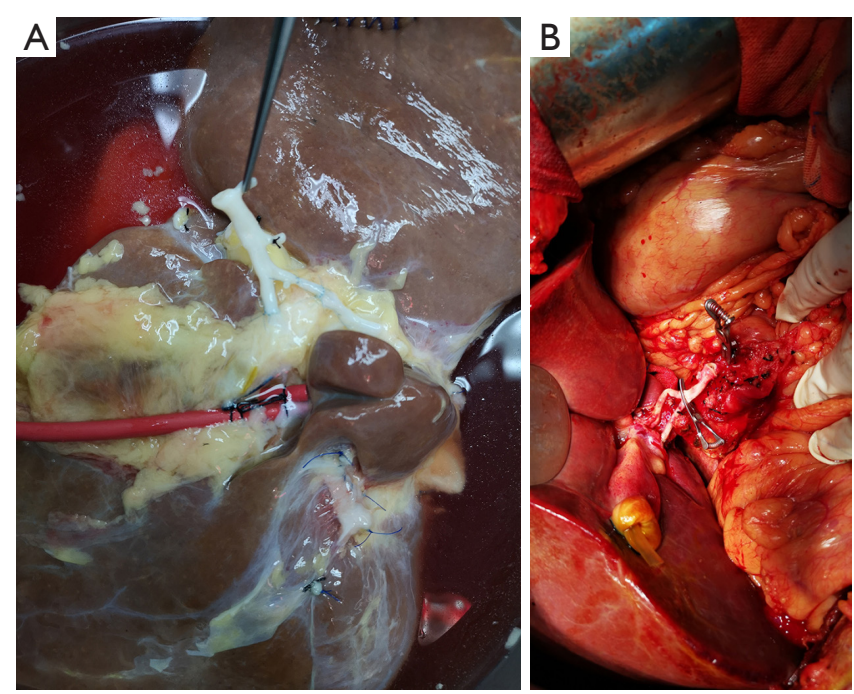

Figure 6 Reconstruction of hepatic artery using superior mesenteric artery and opening the hepatic portal vein. (A) Branches of hepatic artery anastomoses with branches of the superior mesenteric artery. (B) After opening the hepatic portal vein, the superior mesenteric artery was end-to-end anastomosed to the recipient hepatic artery.

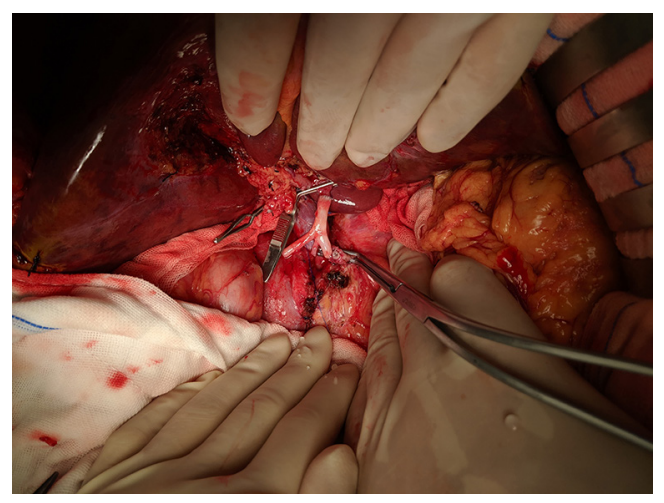

Figure 7 If the common hepatic artery of the donor liver was short, it was first extended with an end-to-end anastomosis of the mesenteric artery in vitro, then end-to-end anastomosed with the recipient hepatic artery.

measured the diameter of the mesenteric artery on CT images. At the level where the left renal vein drains to the inferior vena cava, the mean diameter is $6.5 \mathrm{~mm}$. At the level where the left renal vein drains to the plane $10 \mathrm{~mm}$ above the inferior vena cava, the mean diameter is $6.7 \mathrm{~mm}$. At the level where the left renal vein drains $10 \mathrm{~mm}$ below the inferior vena cava, the diameter is $6.3 \mathrm{~mm}$. The diameter of the SMA 

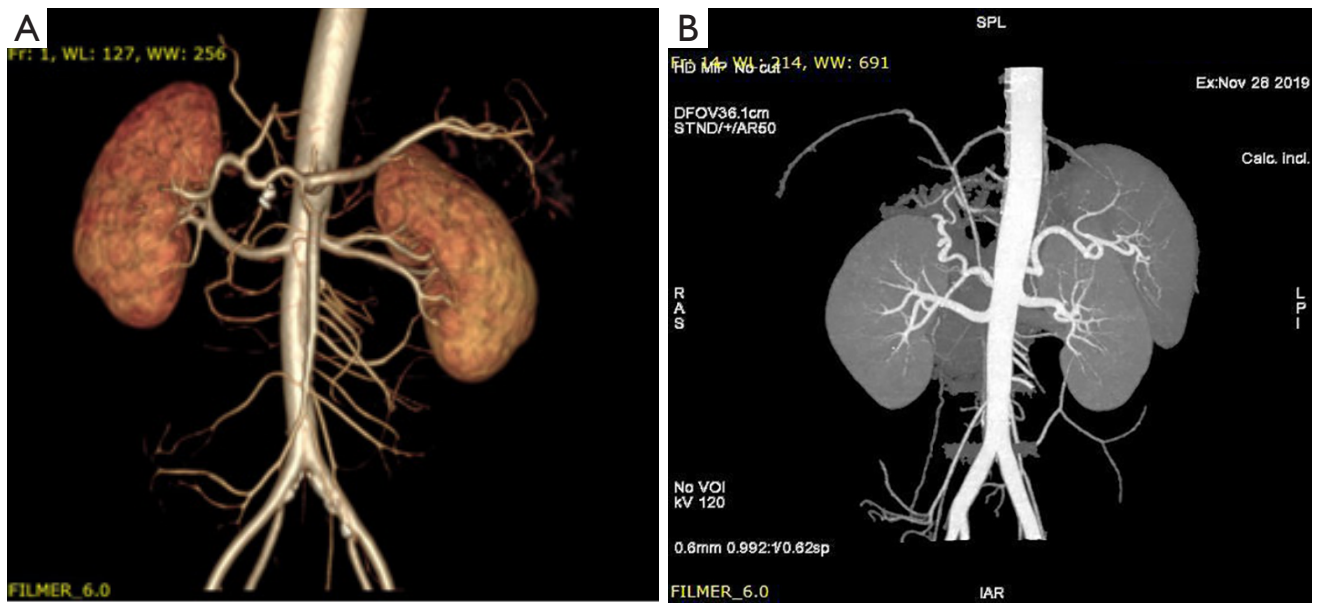

Figure 8 CT angiography (CTA) of the transplanted liver hepatic artery. The anastomosis of hepatic artery was patent, without thrombosis, and the anastomosis has a high matching degree of vascular caliber.

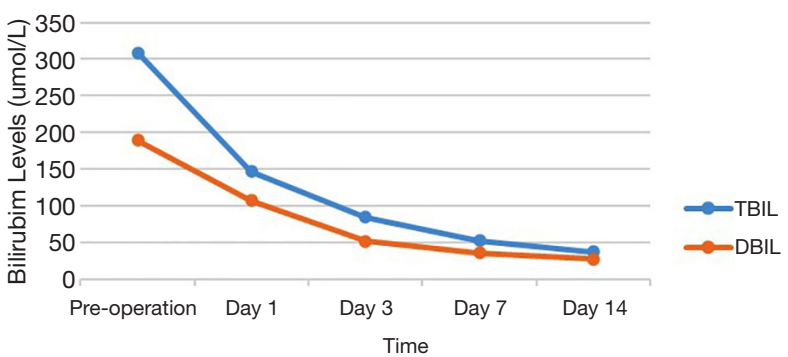

Figure 9 Pre- and post-operative bilirubin levels. TBIL, total bilirubin; DBIL, bilirubin direct.

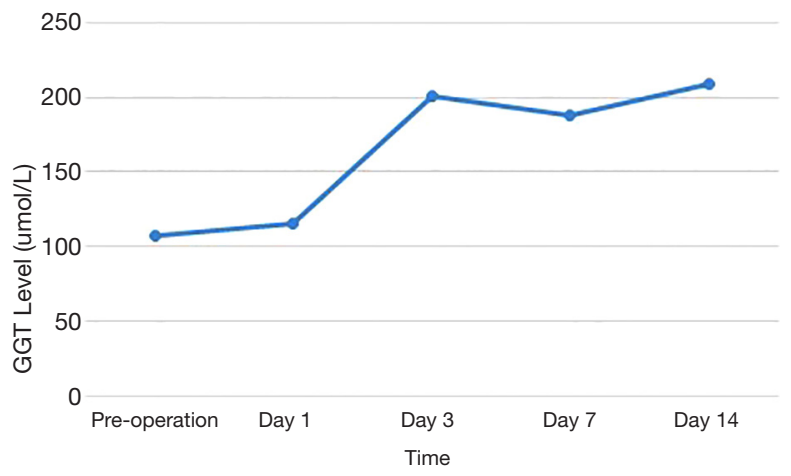

Figure 10 Pre- and post-operative GGT levels. GGT, gammaglutamyl transferase.

branches gradually decreases, and as there are many branches, they can match each hepatic artery or branch with different diameters. It therefore has advantages over the iliac artery in terms of branch matching, and also has good reproducibility.

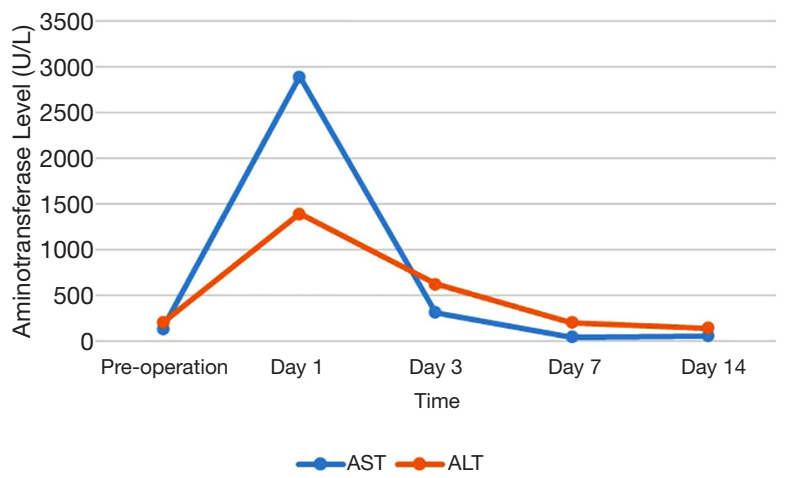

Figure 11 Pre- and post-operative aminotransferase levels. AST, alanine aminotransferase; ALT, aspartate amino transferase.

\section{Conclusions}

Reconstruction of the hepatic artery with the SMA in vitro is a novel method of vascular reconstruction, which ensures blood flow of the hepatic artery, reduces the anastomosis difficulty of the arteries of the donor liver, and reduces the occurrence of vascular complications.

\section{Acknowledgments}

Funding: This work was supported by the Major Clinical Technology Program of Guangzhou, China (grant number 2019ZD12) and Medical Science and Technology Research Foundation of Guangdong Province, China (grant number A2020384). 


\section{Footnote}

Reporting Checklist: The authors have completed the STROBE reporting checklist. Available at http://dx.doi. org/10.21037/atm-20-7200

Data Sharing Statement: Available at http://dx.doi. org/10.21037/atm-20-7200

Conflicts of Interest: All authors have completed the ICMJE uniform disclosure form (available at http://dx.doi. org/10.21037/atm-20-7200). The authors have no conflicts of interest to declare.

Ethical Statement: The authors are accountable for all aspects of the work in ensuring that questions related to the accuracy or integrity of any part of the work are appropriately investigated and resolved. The study was approved by the Second Affiliated Hospital of Guangzhou Medical University (No. ChiCTR1900026543) and written informed consent was obtained from all patients. All procedures performed in this study involving human participants were in accordance with the Declaration of Helsinki (as revised in 2013).

Open Access Statement: This is an Open Access article distributed in accordance with the Creative Commons Attribution-NonCommercial-NoDerivs 4.0 International License (CC BY-NC-ND 4.0), which permits the noncommercial replication and distribution of the article with the strict proviso that no changes or edits are made and the original work is properly cited (including links to both the formal publication through the relevant DOI and the license). See: https://creativecommons.org/licenses/by-nc-nd/4.0/.

\section{References}

1. Silva MA, Jambulingam PS, Gunson BK, et al. Hepatic artery thrombosis following orthotopic liver transplantation: a 10-year experience from a single centre in the United Kingdom. Liver Transpl 2006;12:146-51.

2. Miraglia R, Maruzzelli L, Caruso S, et al. Minimally invasive endovascular and biliary treatments of children with acute hepatic artery thrombosis following liver transplantation. Pediatr Radiol 2014;44:94-102.

3. Pawlak J, Grodzicki M, Leowska E, et al. Vascular complications after liver transplantation. Transplant Proc
2003;35:2313-5.

4. Zheng SS, Liang TB, Yu ZY, et al. Diagnosis and treatment of hepatic artery thrombosis after liver transplantation. Zhonghua Yi Xue Za Zhi 2004;84:1536-40.

5. Hernandez Q, Ramirez P, Munitiz V, et al. Incidence and management of biliary tract complications following 300 consecutive orthotopic liver transplants. Transplant Proc 1999;31:2407-8.

6. Torras J, Lladó L, Figueras J, et al. Biliary tract complications after liver transplantation: type, management, and outcome. Transplant Proc 1999;31:2406.

7. Piardi T, Lhuaire M, Bruno O, et al. Vascular complications following liver transplantation: A literature review of advances in 2015. World J Hepatol 2016;8:36-57.

8. Mourad MM, Liossis C, Gunson BK, et al. Etiology and management of hepatic artery thrombosis after liver transplantation. Liver Transpl 2014;20:713-23.

9. Rajakannu M, Awad S, Ciacio O, et al. Intention-to-treat analysis of percutaneous endovascular treatment of hepatic artery stenosis after orthotopic liver transplantation. Liver Transpl 2016;22:923-33.

10. Yang Y, Zhao JC, Yan LN, et al. Risk factors associated with early and late HAT after adult liver transplantation. World J Gastroenterol 2014;20:10545-52.

11. Herrero A, Souche R, Joly E, et al. Early Hepatic Artery Thrombosis After Liver Transplantation: What is the Impact of the Arterial Reconstruction Type? World J Surg 2017;41:2101-10.

12. Del Gaudio M, Grazi GL, Ercolani G, et al. Outcome of hepatic artery reconstruction in liver transplantation with an iliac arterial interposition graft. Clin Transplant 2005;19:399-405.

13. Warner P, Fusai G, Glantzounis GK, et al. Risk factors associated with early hepatic artery thrombosis after orthotopic liver transplantation-univariable and multivariable analysis. Transpl Int 2011;24:401-8.

14. Chuanbin X, Fagong T, Radiology DO. Anatomical study of the superior mesenteric artery on CT. J Regional Anat Operative Surg 2003;12:8-9.

Cite this article as: Fang J, Chen Z, Jiang N, He Q, Wang S, Zhang W, Ma J, Li G, Zhang L, Xu L, Yin W, Lai X, Chen R, Li L, Liu L, Xiong Y, Zhang T, Xu H, Wan J, Wu J, Pan G, Shi B, Li K. Reconstruction of the hepatic artery using the superior mesenteric artery for liver transplantation. Ann Transl Med 2020;8(22):1520. doi: 10.21037/atm-20-7200 